\title{
Determination of Trace Iron in Three Kinds of Tonic Herbs by Dual-Indicator Dual-Wavelength and Catalytic Kinetic Spectrophotometric Method
}

\author{
Fu Shuang, Lin Qi, Ma Dezhi*, Wang Zhanle \\ Qiqihaer Medical University, Qiqihaer, 161006, China \\ *corresponding author: fsjt1980@163.com
}

Keywords: catalytic spectrophotometry; dual-indicator; dual-wavelength; methyl orange; methylene blue; trace iron.

\begin{abstract}
One catalytic discoloring spectrophotometry with double indicators and dual wavelength is proposed in the Thesis to measure the content of trace iron in three kinds of hematinic, artemisia integrifolia and other vegetables. In HCI medium, methyl orange and methylene blue are indicators. At wavelength of $500 \mathrm{~nm}$ and $665 \mathrm{~nm}$, the change value $\Delta \mathrm{A}$ in absorbance of catalytic system and non-catalytic system is measured and thus the iron content is measured. The conclusion is that the Method is characterized by simple operation, high sensitivity, favorable selectivity and reliable results.
\end{abstract}

\section{Introduction}

Iron is the essential microelement with the highest content in human body and it plays an important role in regulating metabolism of organism. The proportion of iron-deficiency anemia is very high in our country and almost one out of ten suffers from the iron-deficiency anemia. Iron element is the "core element in human body", which almost accounts for $0.006 \%$ of body weight and which has important physiological function and plays an important role in regulating metabolism of human body. In case the storage volume of iron in human body is insufficient, it will influence heme synthesis of cells and thus give rise to anemia ${ }^{[1]}$. There are numerous hematinic in traditional Chinese medicine in which white peony root, Angelica sinensis and polygonum multiflorum are the most common. However, people have dead zone in understanding of traditional Chinese medicine and they think hematinic has high content of iron and thus can be used to supplement trace iron in human body and thus cures anemia without being aware of compatibility of traditional Chinese medicine. As for vegetables, eggplant, artemisia integrifolia and spinach etc. are iron-replenishment foods which are very popular among folks and methods used to test iron content in these drugs and vegetables mainly include atomic absorption spectrophotometry and spectrophotometry. The advantages of atomic absorption spectrophotometry include low detection limit, high sensitivity and mature methods. However, instruments used in this method are expensive in price and tedious in operation and this method is not universal for it requires detection by professionals. As for spectrophotometry, the iron is measured mainly by phen spectrophotometry. The technology is mature but low in sensitivity and thus cannot meet requirements of measuring trace iron. At present, there are some reports of measuring trace iron by spectrophotometry by catalytic kinetics ${ }^{[2,3]}$ but most of methods have single wavelength and single indicator ${ }^{[4]}$ and reports about measuring method with double indicators and dual wavelength are rare. To find out content of iron in traditional Chinese medicine and food, a new detection method is established and the spectrophotometry in which hydrogen peroxide catalyst by iron is used to decolor double indicators such as methyl orange and methylene blue is adopted in the Thesis to measure the trace iron in traditional Chinese medicine and food. Part 1 Experimental 


\subsection{The Instrument and Reagent}

T6 ultraviolet spectrophotometer in the new century (Beijing's general instrument co., LTD.); HH - 6 digital constant temperature water-bath water (guohua electric appliance co., LTD.); AA320NCRT atomic absorption spectrophotometer (Shanghai precision scientific instrument co., LTD.); Electronic universal furnace (suzhou jiangdong precision instruments co., LTD.); Kay type flask (zhengzhou zhongtian experimental instrument co., LTD.).

Radix paeoniae alba, angelica, fleece-flower root, all bought in qiqihaer city pharmacy; Iron (III) standard stock solution: including $100 \mathrm{~g} / \mathrm{mL}$, when diluted into (including $0.1 \mathrm{~g} / \mathrm{mL}$ standard solution; Methyl orange solution: $0.001 \mathrm{~mol} / \mathrm{L}$; Methylene blue: $0.001 \mathrm{~mol} / \mathrm{L}$; Hydrochloric acid: $0.10 \mathrm{~mol} / \mathrm{L}$; Sulfate: $0.05 \mathrm{~mol} / \mathrm{L}$; Hydrogen peroxide: volume fraction of $15 \%$; Concentrated nitric acid (AR); Perchlorate (AR); $\mathrm{NaOH}$ (GR); Secondary distilled water.

\subsection{Experiment Method}

Take two $25 \mathrm{~mL}$ volumetric flask 1 and 2, respectively, in turn, add $0.001 \mathrm{~mol} / \mathrm{L}$ of methyl orange solution $0.80 \mathrm{~mL}, 0.001 \mathrm{~mol} / \mathrm{L} 0.60 \mathrm{~mL}$ methylene blue solution, $0.10 \mathrm{~mol} / \mathrm{L} 1.50 \mathrm{~mL}$ of hydrochloric acid, $15 \%$ hydrogen peroxide $1.00 \mathrm{~mL}$. Adding iron to volumetric flask 2 (III) standard solution $0.30 \mathrm{ml}$ (catalytic systems), water volume, shake well. In 95 plus or minus $0.5^{\circ} \mathrm{C}$ constant temperature water bath pot, heat after $15 \mathrm{~min}$, quickly remove and cooling water for $2 \mathrm{~min}$. With $1 \mathrm{~cm}$ dish, with water as A reference, the wavelength of $500 \mathrm{~nm}$ and $665 \mathrm{~nm}$, respectively measuring catalytic systems (A500 featured, A665), and the catalytic system (A0500, A0665) absorbance, calculate $\Delta \mathrm{A}=(\mathrm{A} 0500$ - A500 featured $)+(\mathrm{A} 0665$ - A665) .

\section{The Results and Discussion}

\subsection{Absorption Curve}

According to section 1.2 of the experimental method, water as the reference, in 300-700 - nm wavelength range to draw the absorption curve of catalysts and catalytic system, the results show that the catalytic system and system at $500 \mathrm{~nm}$ and $665 \mathrm{~nm}$ are absorbing peak, and iron of methyl orange (absorption peak at $500 \mathrm{~nm}$ ) and methylene blue (absorption peak at $665 \mathrm{~nm}$ ) has obvious catalysis, this experiment select measuring wavelength of $500 \mathrm{~nm}$ and $665 \mathrm{~nm}$, as shown in figure 1 .

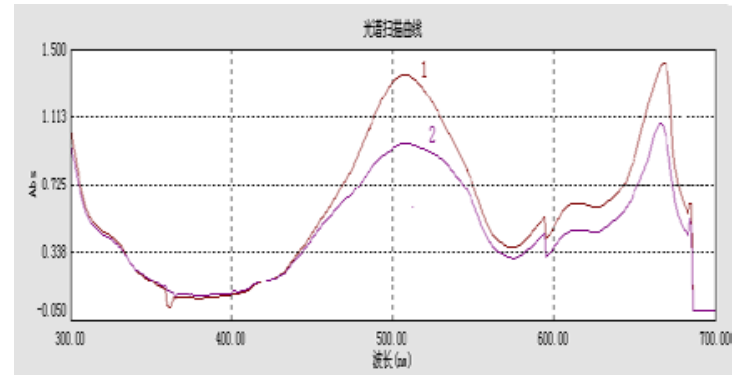

Fig. 1 catalytic systems and the absorption curve of catalytic system

(curve 1: non catalytic system, curve 2: iron catalysts)

\subsection{Reaction Media Choices}

Other conditions according to section 1.2 of the experimental method, fixed and reagent dosage, respectively to investigate $\mathrm{H}_{2} \mathrm{SO}_{4}$ volume $(0.05 \mathrm{~mol} / \mathrm{L})$ to the rightness $\Delta \mathrm{A}$ value, the influence of the experimental results show that: in $\mathrm{HCl}$ medium, the determination sensitivity is higher, so choose $\mathrm{HCl}$ acid medium. 


\subsection{The selection of the amount of hydrochloric acid}

According to the experimental method of section 1.2, and fixed other condition and the dosage of reagents. Adding hydrochloric acid $(0.10 \mathrm{~mol} / \mathrm{L})$ were investigated as the volume of $0.00 、 0.25$ 、 $0.50 、 0.75 、 1.00 、 1.25 、 1.50 、 1.75 、 2.00 \mathrm{~mL}$ for the determination of the value of $\Delta \mathrm{A}$. The experimental results show that: when the amount of $\mathrm{HCl}$ was $1.50 \mathrm{~mL}$, the maximum value in the range of $0.2-0.8$ and the stability of $\Delta \mathrm{A}$. So the selection of hydrochloric acid is $1.5 \mathrm{ml}$ in this experiment. As following, figure 2.

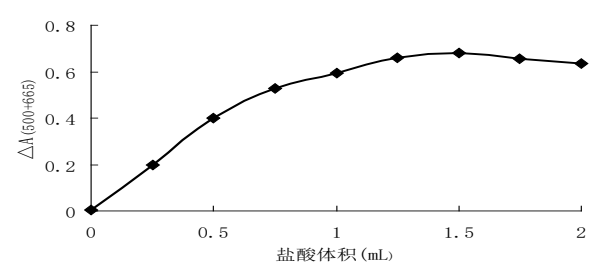

Fig. 2 Effects of the amount of hydrochloric acid on the $\Delta \mathrm{A}$ value.

\subsection{The selection of the amount of Hydrogen peroxide}

According to the experimental method of section 1.2, and fixed other condition and the dosage of reagents. Adding hydrogen peroxide were investigated as the volume of $0.00 、 0.50 、 1.00 、 1.50$ 、 $2.00 、 2.50 、 3.00 \mathrm{~mL}$ for the determination of the value of $\Delta \mathrm{A}$. The experimental results show that: when the amount of $\mathrm{H}_{2} \mathrm{O}_{2}$ was $1.50 \mathrm{~mL}$, the maximum value in the range of $0.2-0.8$ and the stability of $\Delta$ A. So the selection of the amount of $\mathrm{H}_{2} \mathrm{O}_{2}$ is $1.00 \mathrm{ml}$ in this experiment. As following, figure 3 .

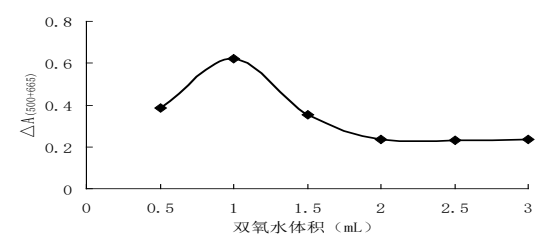

Fig. 3 Effects of the amount of $\mathrm{H}_{2} \mathrm{O}_{2}$ on the $\Delta \mathrm{A}$ value

\subsection{The selection of the amount of Methyl orange solution}

According to the experimental method of section 1.2, and fixed other condition and the dosage of reagents. Adding Methyl orange solution were investigated as the volume from 0 to $1.00 \mathrm{~mL}$, and determining the value of $\Delta \mathrm{A}$ value every $0.10 \mathrm{~mL}$. The experimental results show that: when the amount of Methyl orange solution was $0.80 \mathrm{~mL}$,the maximum value in the range of $0.2-0.8$ and the stability of $\Delta \mathrm{A}$ value. So the selection of the amount of Methyl orange solution is $0.80 \mathrm{ml}$ in this experiment. As following, figure 4.

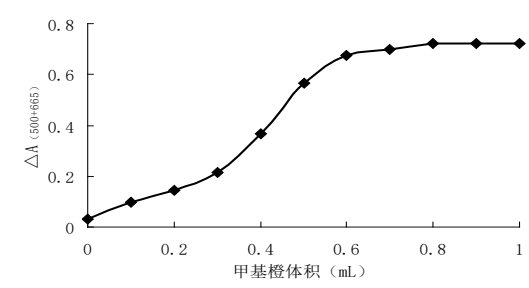

Fig. 4 Effects of the amount of Methyl orange solution on the $\Delta \mathrm{A}$ value.

\subsection{The selection of the amount of Methylene blue}

According to the experimental method of section 1.2, and fixed other condition and the dosage of 
reagents. Adding Methylene blue were investigated as the volume from 0 to $1.00 \mathrm{~mL}$,and determining the value of $\Delta \mathrm{A}$ every $0.10 \mathrm{~mL}$. The experimental results show that: when the amount of Methylene blue was $0.60 \mathrm{~mL}$, the maximum value in the range of $0.2-0.8$ and the stability of $\Delta \mathrm{A}$ value. So the selection of the amount of Methylene blue is $0.60 \mathrm{ml}$ in this experiment. As following, figure 5 .

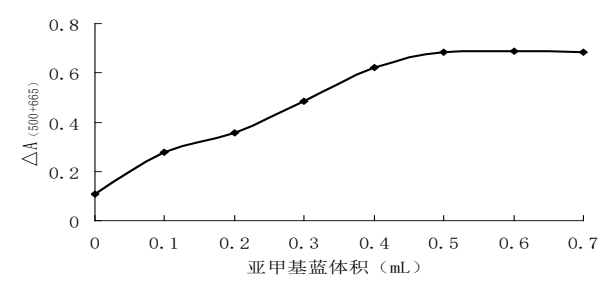

Fig. 5 Effects of the amount of Methylene blue on the $\Delta \mathrm{A}$ value

\subsection{The selection of reaction temperature}

According to the experimental method of section 1.2, and fixed other condition and the dosage of reagents. The influences of reaction temperature were investigated respectively from $70^{\circ} \mathrm{C}$ to $100{ }^{\circ} \mathrm{C}$, and determined $\Delta \mathrm{A}$ value every $5{ }^{\circ} \mathrm{C}$. The experimental results show that: when the reaction temperature was $95^{\circ} \mathrm{C}$, the maximum value in the range of $0.2-0.8$ and the stability of $\Delta \mathrm{A}$ value. So this study selected water bath heating and the temperature is $95{ }^{\circ} \mathrm{C}$.As following, figure 6.

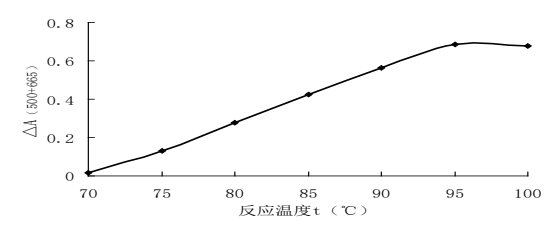

Fig. 6 Effects of the reaction temperature on the $\Delta \mathrm{A}$ value

\subsection{The selection of reaction time.}

According to the experimental methods of section 1.2, and fixed other condition and the dosage of reagents. The influences of reaction time were investigated respectively from 10 to 18min, and and determined $\Delta \mathrm{A}$ value every $1 \mathrm{~min}$. The experimental results show that: when the reaction time was $15 \mathrm{~min}$, the maximum value in the range of $0.2-0.8$ and the stability of $\Delta \mathrm{A}$ value. So this study selected the reaction time is $15 \mathrm{~min}$.As following ,figure 7.

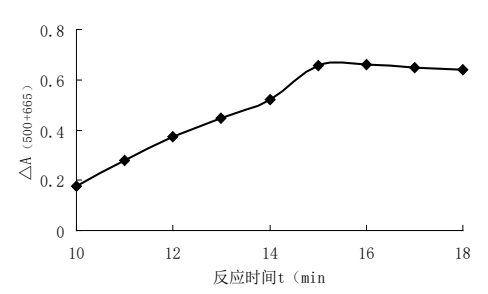

Fig. 7 Effects of the reaction time on the $\Delta \mathrm{A}$ value

\subsection{The working curvy and the detection limit.}

Under the optimum experimental conditions. According to the experimental methods of section 1.2,respectively taking iron standard solution of different experiment. The $\Delta \mathrm{A}$ value were measured at wavelength of $500 \mathrm{~nm}$ and $665 \mathrm{~nm}$.Calculating $\Delta \mathrm{A}$ value and drawing working curve .By working curves we can knoe iron (III) mass concentration showed a good linear 
relationship with the $\Delta \mathrm{A}$ in the range of $0 \sim 0.02000 \mathrm{~g} / \mathrm{mL}$. The linear regression equation is $\Delta \mathrm{A}=$ 38.971 $\rho(\mu \mathrm{g} / \mathrm{mL})-0.0064, \mathrm{r}=0.9995$. By deducted the absorbency of blank value after is 0.01 , and the corresponding concentration as the detection limit. The detection limit of this method that was experimentally measured is $8.0 \times 10-10 \mathrm{~g} / \mathrm{L}$.

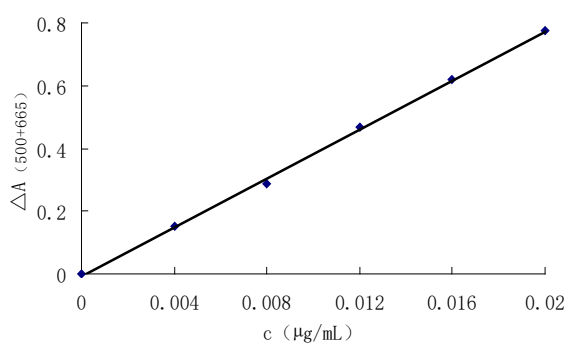

Fig. 8 Working Curve

\subsection{The influence of co-existing ions}

According to the above experimental method, when Fe(III) becoming $0.012 \mu \mathrm{g} / \mathrm{mL}$, testing about 15 kinds of the influence of co-existing ions on the determination results, including the $\mathrm{K}^{+}, \mathrm{Na}^{+}, \mathrm{Ag}^{+}, \mathrm{Cu}^{2+}, \mathrm{CO}_{3}{ }^{2-}, \mathrm{Ca}^{2+}, \mathrm{Cl}^{-}, \mathrm{Mg}^{2+}, \mathrm{Mn}^{2+}, \mathrm{Zn}^{2+}$ and so on ,this relative error of plus or minus $5 \%$ or less, the results showed that in addition to the $\mathrm{Cu}^{2+}$ allowances for 1 times, 1000 times the rest of the sample quantity will not affect the measurement. Therefore, most of the ion of allowances is higher, general samples can be directly determined.

\subsection{The analysis of sample}

Respectively measure $0.5 \mathrm{~g}$ of dry and smashed white peony root, Angelica sinensis and polygonum multiflorum as well as mixture of dry and smashed eggplant peel, pulp and stalk, mixture of neck and leaf of artemisia integrifolia and mixture of neck and leaf of spinach, and then add $10 \mathrm{~mL}$ perchloric acid and $10 \mathrm{~mL}$ concentrated nitric acid to $250 \mathrm{~mL}$ Kjeldahl flask and place it for 24h. And then, it is placed in electronic multi-purpose furnace for digestion until it turns into colorless and transparent solution. Adjust the $\mathrm{pH}$ value to be 5 and then set the constant volume to be $100 \mathrm{~mL}$ and then take some solution to measure according to experimental method. Compare the result with results measured by atomic absorption spectrophotometry and the experiment results show that results of the method are reliable and its accuracy and recovery rate conform to requirements.

Table 1 Sample analysis results

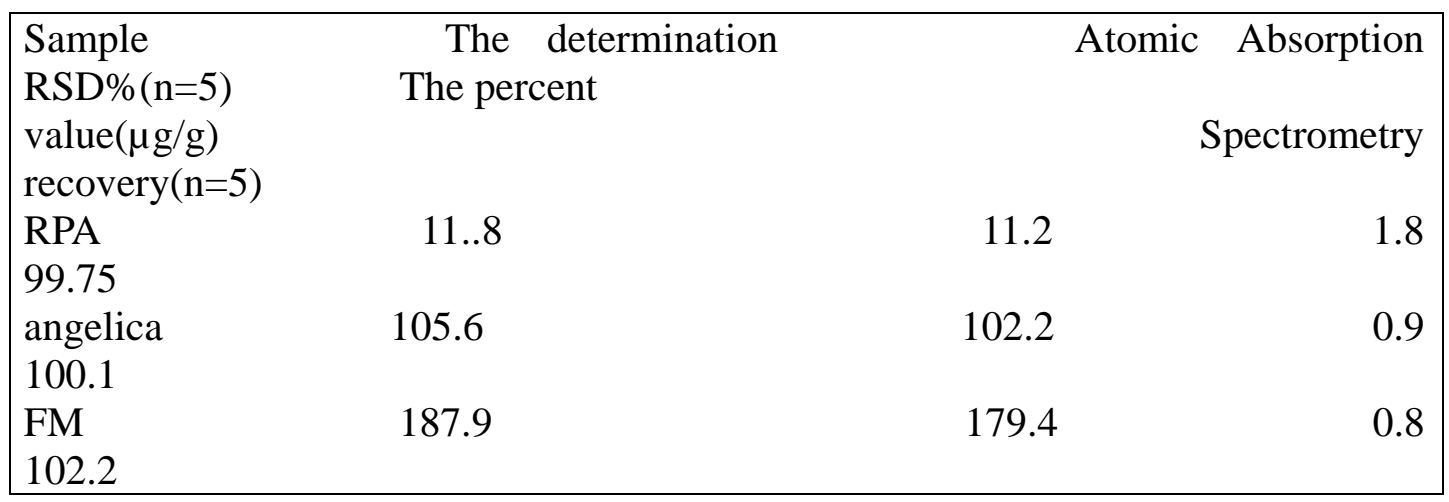


Table 2 Test results of iron content in vegetable samples

\begin{tabular}{|c|c|c|c|}
\hline Sample & The method value $(\mu \mathrm{g} / \mathrm{g})$ & Atomic absorption & $\mathrm{RSD} \%(\mathrm{n}=5)$ \\
\hline $\begin{array}{l}\text { Rate of recc } \\
(n=5)\end{array}$ & ery\% & spectrophotometry & \\
\hline $\begin{array}{l}\text { eggplant } \\
100.5\end{array}$ & 904.4 & 898.2 & 1.5 \\
\hline $\begin{array}{l}\text { Artemisia } \\
99.7\end{array}$ & 113.5 & 106.2 & 1.2 \\
\hline $\begin{array}{l}\text { Spinach } \\
101.5\end{array}$ & 7.533 & 8.235 & 0.9 \\
\hline
\end{tabular}

\section{Discussion}

In $\mathrm{HCl}$ medium is presented in this paper, the iron catalyzed hydrogen peroxide oxidation of methyl orange and methylene blue indicator fade new method for determination of trace iron. The two using methylene blue and methyl orange indicator method for the determination of double wavelength has not yet been reported, the method for determining high sensitivity low detection limit good selectivity , the result is reliable, suitable for the determination of trace concentrations of iron.

\section{Acknowledgements}

Fund Project: College Students Innovation and Entrepreneurship Training Program of Heilongjiang Province (201511230024);

Fund Project of Qiqihar Medical College (QY2016Z-06)

Corresponding author: Ma Dezhi (1965) male, professor, Research area: extraction of effective components in food and traditional Chinese medicine. E-mail: fsjt1980@163.com

\section{References}

[1] Li Rongyu. Five kinds of Chinese herbal medicine fe content determination and analysis of J.China's health care nutrition 2012: 2191-2192

[2] Yan-xia li, ya-hong Chen. Suppression of ethyl orange faded kinetic spectrophotometric determination of trace iron (III) [J].Journal of Analytical Science 2013,29(2): 288-290

[3] Bahr guli. buick tursun. Adjacent phenanthroline colorimetry analysis the primeval forest soil fe content [J]. Chinese Journal of Spectroscopy Laboratory 2013, 30 (5) : 2314-2318

[4] Huang Hui, Xie Xia feng, etc.Solid phase spectrophotometry determination of trace iron [J]. Journal of zhejiang normal university (natural science edition), 2004, 27 (3) : 272-275

[5] Tomiyasu T, Sakamoto H , Yonehara N. Catalytic determination of iron by a fixed-time method using the oxidation reaction of chlorpromazine with hydrogenperox ide[J] . Anal. Sci, 1996, 12 ( 3) : 507-509

[6] Susumu K,Yuki H , Yukiko H, et al. Speciation of iron in river and tap waters by catalytic Spectrophotometry using oxidatio n of phenglenediamine with hydrogenperox ide[ J].Anal. Chim. Acta, 1999, 388: 35-43

[7] Jian-hua xing,Jian-hao Xin, Zhi-gang Guo, etc. Catalytic kinetic spectrophotometric determination of trace iron in plant protein beverage [J]. The physical and chemical inspection (chemical volume):2012,48( 6) : 731-734. 
[8] Xian-yi Shi,Xiao-qing Wu,etc. Hydrogen peroxide - o-aminophenol system catalytic chromogenic spectrophotometric method of determination of trace iron $[\mathrm{J}]$. Chinese Journal of Spectroscopy Laboratory,2011,28 (2) : 711-714

[9] Xiao-lan Ge,Xiu-juan Xie,etc. Double wavelengths double indicator catalytic kinetic spectrophotometric determination of trace iron [J]. Chemical Research and ApplicationChem Res Appl，2010，22（2） : 191-193

[10] Deng-ming Sn*,Qing-ren Zhu,etc. Double wavelengths double indicator catalytic kinetic spectrophotometric determination of trace iron [J]. Metallurgical Analysis, 2007, 27 (1) : 42-44 